$\mathrm{p}=0.02)$ comorbidities increased over time in ACPA negatives and remained stable in ACPA+ (CV: 33.0, 30.8, 40.7, NS; cancer: 3.0, 4.4, 6.2, NS). RF positivity decreased by period $(47.8,36.9,36.7 \%, p=0.03)$, but ACPA+ remained stable $(40.8,35,35.4 \% \mathrm{NS})$.

Outcomes over 5 years of follow up.

There was no link between the presence of any biomarker and subsequent disease activity scores. Positive ACPA, RF and anti-Sa at baseline predicted development of more erosive status $(R R=1.50 ; 1.37$ and 1.52 , all $p<0.001)$. $68 \%$ reached DAS28 remission overall $(70.3 \%$ in ACPA negatives, $65.2 \%$ in ACPA+). Independent of ACPA status, remission rates increased between Periods 1 vs $2(R R=1.14, p=0.04)$ and 1 vs $3(R R=1.13, p=0.055)$, but not between 2 vs 3 . CV comorbidities among ACPA+ increased significantly more over time vs ACPA negatives $(+8.9 \%$ vs $+4.1 \%$; $R R=1.18, p=0.03)$. Erosion scores increased significantly more in ACPA+ treated with DMARDs only vs receiving a biologic ( $\Delta$ Sharp: 3.98 vs $3.11, p=0.026 ; \Delta$ Erosions: 2.58 vs 1.83 , $p=0.02$ ). By period, erosive status decreased significantly (Periods 2 vs $1: R R=$ $0.65, p=0.002$; 3 vs 1 : $R R=0.42, p=0.002 ; 3$ vs 2 : $R R=0.64, p=0.007$ ), both in $\mathrm{ACPA}+$ and negatives.

Conclusion: In this cohort of recent onset $\mathrm{RA}$ recruited over 20 years, we observed a constant drift towards RF-negative arthritis at baseline with decreasing smoking rates and increasing comorbidities in seronegative patients. Positive antibodies were associated more cardiovascular comorbidities accrual. Autoantibody positive (especially ACPA+) patients developed more erosive disease and had better erosion outcomes with biologic treatments. Irrespective of the presence or absence of biomarkers, marked improvements in control of disease activity and in remission rates occured over each period.

References:

[1] Boire G, et al. Arthritis Res Ther 2005;7:R592-R603;

[2] Carrier N, et al. Arthritis Res Ther 2016;18:37

Disclosure of Interests: Nathalie Carrier: None declared, Sophie Roux: None declared, Ariel Masetto: None declared, Artur J. deBrum Fernandes: None declared, Patrick Liang: None declared, Meryem Maoui Employee of: Bristol Myers Squibb Canada, Gilles Boire Grant/research support from: Merck Canada (Registry of biologices, Improvement of comorbidity surveillance)

Amgen Canada (CATCH, clinical nurse)

Abbvie (CATCH, clinical nurse)

Pfizer (CATCH, Registry of biologics, Clinical nurse)

Hoffman-LaRoche (CATCH)

UCB Canada (CATCH, Clinical nurse)

BMS (CATCH, Clinical nurse, Observational Study Protocol IM101664. SEROPOSITIVITY IN A LARGE CANADIAN OBSERVATIONAL COHORT)

Janssen (CATCH)

Celgene (Clinical nurse)

Eli Lilly (Registry of biologics, Clinical nurse), Consultant of: Eli Lilly, Janssen, Novartis, Pfizer, Speakers bureau: Merck, BMS, Pfizer

DOI: 10.1136/annrheumdis-2020-eular.717

\section{FRI0032 REGIONAL AND WIDESPREAD PATTERNS OF NON-ARTICULAR PAIN ARE COMMON AT RA DIAGNOSIS AND CONTRIBUTE TO POOR OUTCOMES AT 12 MONTHS: A PROSPECTIVE STUDY OF PAIN PATTERNS IN CANADIANS WITH RA}

V. Bykerk ${ }^{1}$, O. Schieir ${ }^{2}$, M. F. Valois ${ }^{3}$, L. Bessette ${ }^{4,5}$, G. Boire ${ }^{6}$, G. Hazlewood ${ }^{7}$, C. Hitchon ${ }^{8}$, E. Keystone ${ }^{2}$, D. Tin ${ }^{9}$, C. Thorne ${ }^{9}$, J. Pope ${ }^{10}$, S. J. Bartlett ${ }^{3}$ on behalf of CATCH Investigators. ${ }^{1}$ Hospital for Special Surgery, New York, United States of America; ${ }^{2}$ University of Toronto, Toronto, Canada; ${ }^{3}$ Mc Gill University, Montreal, Canada; ${ }^{4}$ Laval University and $\mathrm{CHU}$ de Quebec, Quebec City, Canada; ${ }^{5}$ Centre de l'Ostéoporose et de Rhumatologie de Québec, Department of Medicine,
Quebec City, Canada; ${ }^{6}$ University of Sherbrooke, Faculty of Medicine and Health Sciences, Sherbrooke, Canada; ${ }^{7}$ University of Calgary, Calgary, Canada; ${ }^{8}$ University of Manitoba, Winnipeg, Canada; ${ }^{9}$ Southlake Regional Health Center, Newmarket, Canada; ${ }^{10}$ University of Western, London, Canada

Background: Persistent pain can occur in early RA patients, despite improvement in synovitis and may be due to coexisting non-articular pain (NAP). Though NAP is often attributed to fibromyalgia and widespread NAP, regional NAP syndromes may be more common and under-recognized.

Objectives: To describe patterns of NAP, predictors of persistent NAP and impact on outcomes in the first year following early RA diagnosis.

Methods: Data were from participants enrolled in the Canadian Early Arthritis Cohort (CATCH) between 2017-2019 who completed 0,6,12-month evaluations with patient-reported outcomes [PROs] and clinical data available. We used the McGill Body Pain Diagram (BPD) to classify patients as experiencing no NAP, regional (RP:1-2 regions) or widespread NAP (WP:3-5 regions). Multinomial regression was used to identify baseline predictors of persistent RP and WP at 12-months. Multi-adjusted GEE with linear and logit links were used to estimate time-varying associations of NAP patterns with outcomes updated at each time point.

Results: Study included 421 participants: $66 \%$ were female, with a mean(sd) age 56 (14); $72 \%$ were seropositive and $90 \%$ were treated with MTX \pm csDMARDs as initial therapy. NAP at baseline was common (55\%), with majority (62\%) reporting regional NAP. NAP prevalence was $33 \%$ at 12 months (Figure). Female sex and baseline depressive symptoms were independent predictors of widespread NAP at 12 months while poorer function and lack of early MTX treatment independently predicted regional NAP, at 12 mos. Regional and widespread NAP were associated with lower likelihood of remission in adjusted models that accounted for changes in NAP and remission over time (Table).

Conclusion: NAP is commonly reported in early RA pts seen in real world settings. Regional NAP was more common than WSP at all time-points, but both NAP patterns were associated lower odds of achieving remission targets by 12 months. These data support considering the role of NAP when assessing RA treatment efficacy during clinical visits and warrant different treatment approaches to reduce symptoms in RA patients receiving target-based care.

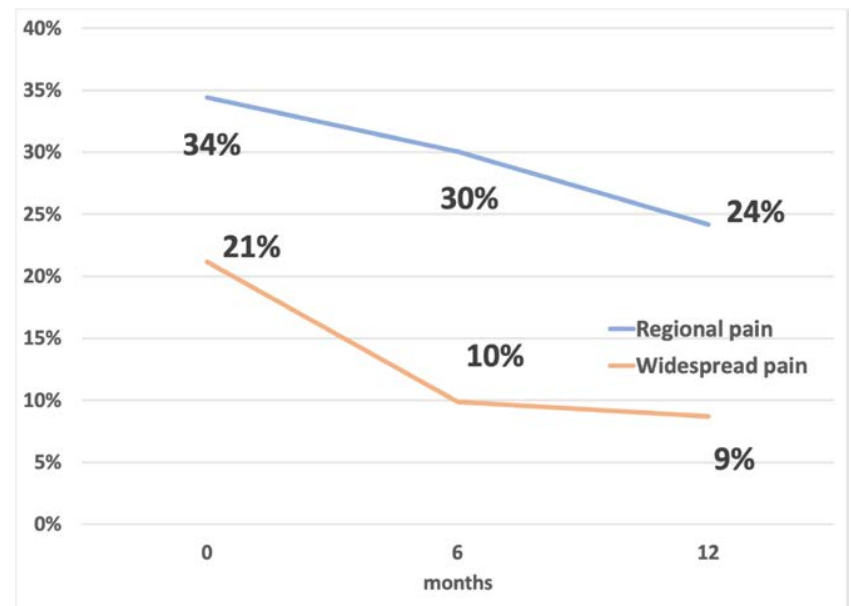

Figure. Point prevalence of regional and widespread NAP at baseline, 6 and 12 months.

Table. Results of Multi-Adjusted GEE Logistic Regression showing Regional and Widespread NAP is associated with a reduced likelihood of achieving Stringent Remission Targets

Boolean Remission Outcome

Disease stage and Clinical Disease Activity

NAP Pattern by Body Pain Diagram Regional vs No NAP Widespread vs No NAP Age

Women vs Men

$\mathrm{RDCl}^{\phi}$ at baseline

Symptom duration

Seronegative vs ACPA+/RF

MTX in first 3 months

Oral Steroids in first 3

months
$0.34(0.18,0.66)$

$0.21(0.07,0.67)$

$\mathrm{NI}$

$\mathrm{N}$

$\mathrm{NI}$

NI

$\mathrm{NI}$

SDAI Remission Outcome

\begin{tabular}{cccc}
\hline $\begin{array}{c}\text { Age/sex adjusted } \\
\text { OR }(95 \% \mathrm{Cl})\end{array}$ & Fully & Age/sex adjusted & Fully Adjusted \\
& Adjusted & OR $(95 \% \mathrm{Cl})$ & \\
\hline
\end{tabular}

$\mathbf{0 . 4 1}(\mathbf{0 . 2 0 , 0 . 8 3 )}$
$\mathbf{0 . 2 7}(\mathbf{0 . 0 8 , 0 . 8 5 )}$
$1.01(0.98,1.04)$
$0.76(0.41,1.42)$
$0.91(0.73,1.14)$
$0.96(0.86,1.08)$
$1.15(0.60,2.19)$
$1.51(0.73,3.13)$
$0.53(0.25,1.10)$

$0.41(0.23,0.71)$

$0.23(0.09,0.61)$

$\mathrm{NI}$

$\mathrm{NI}$

$\mathrm{NI}$

$\mathrm{NI}$

$\mathrm{NI}$
$0.55(0.30,1.00)$

$0.29(0.11,0.80)$

$1.01(0.98,1.03)$

$0.69(0.37,1.27)$

$0.90(0.74,1.10)$

$1.00(0.90,1.10)$

$1.43(0.79,2.60)$

$1.20(0.64,2.24)$

$0.44(0.22,0.87)$

${ }^{\phi} \mathrm{RDCl}=$ rheumatic disease comorbidity index 
Disclosure of Interests: Vivian Bykerk: None declared, Orit Schieir: None declared, Marie-France Valois: None declared, Louis Bessette Grant/research support from: AbbVie, Amgen, Bristol-Myers Squibb, Celgene, Eli Lilly, Janssen, Merck, Novartis, Pfizer, Roche, Sanofi, UCB Pharma, Consultant of: AbbVie, Amgen, Bristol-Myers Squibb, Celgene, Eli Lilly, Janssen, Merck, Novartis, Pfizer, Roche, Sanofi, UCB Pharma, Speakers bureau: AbbVie, Amgen, Bristol-Myers Squibb, Celgene, Eli Lilly, Janssen, Merck, Novartis, Pfizer, Sanofi, Gilles Boire Grant/research support from: Merck Canada (Registry of biologices, Improvement of comorbidity surveillance)

Amgen Canada (CATCH, clinical nurse)

Abbvie (CATCH, clinical nurse)

Pfizer (CATCH, Registry of biologics, Clinical nurse)

Hoffman-LaRoche (CATCH)

UCB Canada (CATCH, Clinical nurse)

BMS (CATCH, Clinical nurse, Observational Study Protocol IM101664. SEROPOSITIVITY IN A LARGE CANADIAN OBSERVATIONAL COHORT) Janssen (CATCH)

Celgene (Clinical nurse)

Eli Lilly (Registry of biologics, Clinical nurse), Consultant of: Eli Lilly, Janssen, Novartis, Pfizer, Speakers bureau: Merck, BMS, Pfizer, Glen Hazlewood: None declared, Carol Hitchon Grant/research support from: UCB Canada; Pfizer Canada, Edward Keystone Grant/research support from: AbbVie, Amgen, Bristol-Myers Squibb, F. Hoffmann-La Roche Inc, Gilead, Janssen Inc, Lilly Pharmaceuticals, Pfizer Pharmaceuticals, Sanofi-Aventis, Consultant of: AbbVie, Amgen, AstraZeneca Pharma, Biotest, Bristol-Myers Squibb Company, Celltrion, Crescendo Bioscience, F. Hoffmann-La Roche Inc, Genentech Inc, Gilead, Janssen Inc, Lilly

Pharmaceuticals, Merck, Pfizer Pharmaceuticals, Sandoz, UCB., Speakers bureau: Amgen, AbbVie, Bristol-Myers Squibb Canada, F. Hoffmann-La Roche Inc., Janssen Inc., Merck, Pfizer Pharmaceuticals, Sanofi Genzyme, UCB, Diane Tin: None declared, Carter Thorne Consultant of: Abbvie, Centocor, Janssen, Lilly, Medexus/Medac, Pfizer, Speakers bureau: Medexus/ Medac, Janet Pope Grant/research support from: AbbVie, Bristol-Myers Squibb, Eli Lilly \& Company, Merck, Roche, Seattle Genetics, UCB, Consultant of: AbbVie, Actelion, Amgen, Bayer, Boehringer Ingelheim, Bristol-Myers Squibb, Eicos Sciences, Eli Lilly \& Company, Emerald, Gilead Sciences, Inc., Janssen, Merck, Novartis, Pfizer, Roche, Sandoz, Sanofi, UCB, Speakers bureau: UCB, Susan J. Bartlett Consultant of: Pfizer, UCB, Lilly, Novartis, Merck, Janssen, Abbvie, Speakers bureau: Pfizer, UCB, Lilly, Novartis, Merck, Janssen, Abbvie

DOI: 10.1136/annrheumdis-2020-eular.3902

\section{FRI0033 ANTI-CARBAMYLATED PROTEIN POSITIVITY PREDICTS DAS28-REMISSION AT 12 MONTHS IN PATIENTS WITH EARLY RHEUMATOID ARTHRITIS: RESULTS FROM THE SINGAPORE EARLY ARTHRITIS COHORT}

J. Cho $^{1}$, A. Mak ${ }^{2}$, S. Agrawal ${ }^{2}$, P. Dhanasekaran ${ }^{3}$, L. K. Teoh ${ }^{4}$, P. Cheung ${ }^{2}$ M. Lahiri ${ }^{2} .{ }^{1}$ National University Hospital, Singapore, Singapore; ${ }^{2}$ National University Hospital, Singapore, Singapore; ${ }^{3}$ National University of Singapore, Singapore, Singapore; ${ }^{4}$ National University Hospital, Singapore, Singapore

Background: Anti-carbamylated protein antibody (anti-carp) positivity has been associated with poorer outcomes in Western cohorts of early rheumatoid arthritis; however, it is unknown if this applies to Asians.

Objectives: We determined whether anti-carp predicted DAS28-remission, disability and radiographic progression in a multi-ethnic Asian ERA cohort.
Methods: Patients with physician diagnosed ERA (symptom duration $\leq 1$ year) were recruited from the Singapore Early Arthritis Cohort $(n=317)$ by convenience sampling. Serum anti-carp was measured cross-sectionally using a commercial ELISA (SincereBio). The test was repeated in 40 healthy individuals to establish the optimal sensitivity and specificity for the diagnosis of RA via a receiver operating curve. Disease activity (DAS28-ESR or DAS28-CRP) was recorded at baseline, 3, 6 and 12 months. Two independent accessors quantified the radiographic damage at baseline and at follow-up using the modified Sharp van der Heijde score (mSS). We used multivariable logistic regression to determine whether anti-carp predicted the following outcomes; (i) DAS-28 remission at 12 months, (ii) any disability (mHAQ>0) at 12 months and (iii) radiographic progression (any increase in the $\mathrm{mSS}$ ). In each regression model, we chose covariates known to influence the dependent variable in our cohort or from literature.

Results: One hundred patients were recruited, of mean age (SD) 49.8 (12.5) years, median (IQR) disease duration 10.2 (6.9-15.1) weeks at cohort entry and baseline median DAS-28 4.5 (2.9-5.9) (Table 1). The anti-carp assay was performed after a median (IQR) disease duration of 2.24 (1.82-3.14) years. 93 patients had baseline hand radiographs and 66 had follow-up hand radiographs after $\geq 12$ months. Receiver operating characteristics curve yielded optimal sensitivity $(95 \%)$ and specificity $(60 \%)$ for the diagnosis of RA at 1.600 D. Therefore, 60 patients were anti-carp positive and 35 patients $(37.2 \%)$ were positive for RF, ACPA and anti-carp (Figure 1). Anti-carp positivity independently predicted DAS28-remission at 12 months (OR 3.41, 95\% Cl 1.08-10.7, $p=0.04$ ) (Table 2) Anti-carp positivity did not predict disability at 12 months (OR $0.61,95 \% \mathrm{Cl} 0.18$ $2.07, p=0.43$ ) or radiographic progression (OR $0.23,95 \% \mathrm{Cl} 0.03-2.03, p=0.18$ )

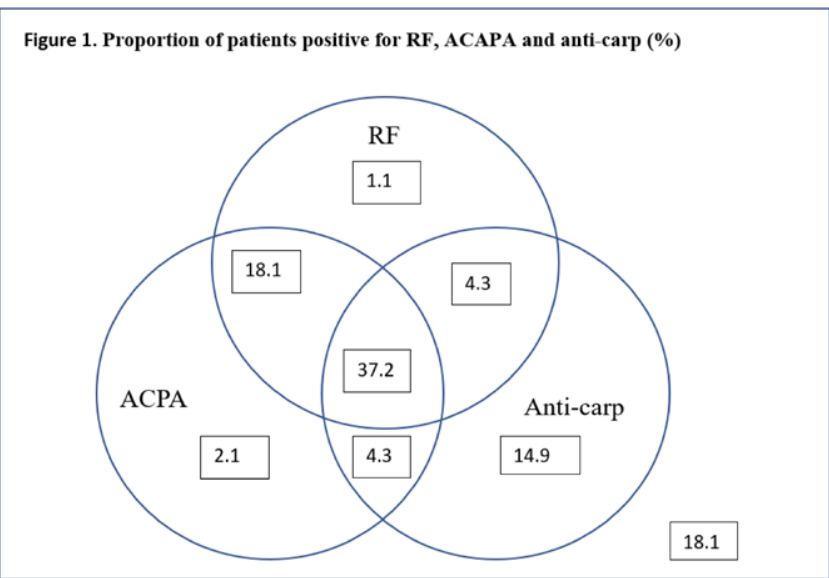

Conclusion: Contrary to previous studies done on Western cohorts where anti-carp predicted worse outcomes, anti-carp positivity predicted DAS28-remission at 12 months in our multi-ethnic Asian cohort. This suggests that differ ent genetic and environmental determinants account for anti-carp expression in patients with RA.

Disclosure of Interests: Jiacai Cho: None declared, Anselm Mak Speakers bureau: Professor Anselm Mak has been paid as a speaker for Johnson \& Johnson., Sachin Agrawal: None declared, Preeti Dhanasekaran: None declared Lay Kheng Teoh: None declared, Peter Cheung: None declared, Manjari Lahiri Grant/research support from: Manjari Lahiri is the site principal investigato for the Singapore National Biologics Register, which is a multi-pharmaceutical funded register, in which industry sponsors provide support through the

Table 1. Predictors of DAS28-remission at 12 months

\begin{tabular}{|c|c|c|c|c|c|c|c|}
\hline \multirow[b]{2}{*}{ Variable } & & \multirow[b]{2}{*}{$N(\%)$} & \multicolumn{2}{|c|}{ Univariable Logistic Regression } & \multicolumn{3}{|c|}{ Multivariable Logistic Regression } \\
\hline & & & OR & $p$ & $\mathrm{OR}(\mathrm{Cl})$ & $S E$ & $p$ \\
\hline Anti-carp & & $60(60)$ & $3.0(1.31-6.88)$ & 0.01 & $3.41(1.08-10.7)$ & 1.99 & 0.04 \\
\hline \multirow[t]{3}{*}{ Serology } & RF and ACPA negative & $31(33.0)$ & Ref & & & & \\
\hline & Either RF or ACPA positive & $11(11.7)$ & $0.99(0.25-3.93)$ & 0.99 & $1.10(0.17-7.04)$ & 1.04 & 0.92 \\
\hline & RF and ACPA positive & $52(55.3)$ & $1.12(0.45-2.75)$ & 0.80 & $0.89(0.28-2.81)$ & 0.52 & 0.84 \\
\hline \multirow[t]{4}{*}{ Baseline DAS28 } & Remission & $17(17.4)$ & Ref & & & & \\
\hline & Low DA & 10 (10.2) & $0.50(0.05-4.67)$ & 0.54 & $0.13(0.01-1.67)$ & 0.17 & 0.12 \\
\hline & Mod DA & $32(32.7)$ & $0.29(0.05-1.65)$ & 0.16 & $0.10(0.02-0.68)$ & 0.10 & 0.02 \\
\hline & High DA & $39(39.8)$ & $0.18(0.04-0.90)$ & 0.04 & $0.06(0.01-0.41)$ & 0.06 & $<0.01$ \\
\hline \multicolumn{2}{|c|}{ Combination csDMARDs or biologic DMARD } & $74(74)$ & $1.13(0.46-2.76)$ & 0.80 & $1.97(0.58-6.67)$ & 1.23 & 0.28 \\
\hline \multicolumn{2}{|c|}{ Radiographic damage at baseline } & $11(20)$ & $1.79(0.65-4.95)$ & 0.26 & $1.27(0.33-4.95)$ & 0.88 & 0.73 \\
\hline \multicolumn{2}{|l|}{ Tertiary education } & $23(38.3)$ & $0.77(0.34-1.77)$ & 0.54 & $0.42(0.12-1.45)$ & 0.27 & 0.17 \\
\hline \multirow[t]{3}{*}{ Ethnicity } & Chinese & $42(70)$ & Ref & & & & \\
\hline & Malay & $39(68.4)$ & $0.61(0.22-1.71)$ & 0.35 & $0.56(0.14-2.25)$ & 0.40 & 0.41 \\
\hline & Indian & $8(13.3)$ & $0.60(0.20-1.77)$ & 0.35 & $0.79(0.20-3.13)$ & 0.56 & 0.74 \\
\hline \multicolumn{2}{|l|}{ Females } & $46(76.7)$ & $0.43(0.17-1.11)$ & 0.08 & $0.48(0.13-1.85)$ & 0.33 & 0.29 \\
\hline
\end{tabular}

